\title{
Different methods of dental caries diagnosis in an epidemiological setting
}

\author{
Renato Pereira da Silva ${ }^{1}$, Andréa Videira Assaf ${ }^{2}$, Gláucia Maria Bovi Ambrosano ${ }^{3}$, Fábio Luiz Mialhe ${ }^{4}$, \\ Marcelo de Castro Meneghim ${ }^{5}$, Antonio Carlos Pereira ${ }^{5}$
}

\author{
${ }^{1}$ Universidade Federal de Viçosa - UFV, Department of Nutrition and Health, Area of Public Health, Viçosa, MG, Brazil. \\ ${ }^{2}$ Universidade Federal Fluminense - UFF, Dental School, Department of Specific Formation, Nova Friburgo, RJ, Brazil \\ ${ }^{3}$ Universidade Estadual de Campinas - UNICAMP, Piracicaba Dental School, Department of Community and Preventive Dentistry, Area of Biostatistics, \\ Piracicaba, SP, Brazil. \\ ${ }^{4}$ Universidade Estadual de Campinas - UNICAMP, Piracicaba Dental School, Department of Community and Preventive Dentistry, Area of Health Education, \\ Piracicaba, SP, Brazil. \\ ${ }^{5}$ Universidade Estadual de Campinas - UNICAMP, Piracicaba Dental School, Department of Community and Preventive Dentistry, Area of Preventive \\ Dentistry and Public Health, Piracicaba, SP, Brazil.
}

\begin{abstract}
Aim: To evaluate the performance of dental caries detection when adjunct methods are associated and their applicability in epidemiological survey of dental caries, at D3 (cavitated carious lesions at dentin layer) and D1+D3 (non cavitated and cavitated carious lesions at enamel/dentin layer) diagnostic thresholds. Methods: A total of 2189 posterior teeth from 165 12-year-old schoolchildren underwent visual examination without (CL1) and with artificial lighting (CL2), radiographic bitewing (BW), fiber-optic transillumination/FOTI (FT), DIAGNOdent ${ }^{\mathrm{TM}}(\mathrm{DD})$ and associations of these methods. Reproducibility was calculated by Kappa statistics and validity was calculated by sensitivity, specificity and accuracy tests. ANOVA (Scott-Knott test) was performed in order to compare the average values of DMF-S obtained by the diagnostic methods. Results: The CL2FTDDBW (D3) and CL2BW (D1+D3) exams presented the highest values for accuracy at epidemiological setting. The DMF-S index obtained for those exams was statistically different at D3 and D1+D3 thresholds. Conclusions: The association of adjunct methods increased the validity of dental caries examination in an epidemiological setting. However, the potential of CL2BW (traditional caries detection methods) or visual exam performed under a more refined diagnostic criteria must be considered in dental caries epidemiological surveys.
\end{abstract}

Keywords: dental caries; diagnosis, oral; epidemiology.

\section{Introduction}

Received for publication: January 23, 2015 Accepted: March 23, 2015

Correspondence to: Renato Pereira da Silva Universidade Federal de Viçosa Departamento de Nutrição e Saúde Av. Peter Henry Rolfs, s/n 36570-900, Viçosa, MG, Brazil Phone: +55 3138992545 Fax: +55 3138992108 E-mail: renatop.silva@ufv.br
With the purpose of improving the quality of dental caries diagnosis, the association of adjunct diagnostic methods with the conventional visual examination has been proposed, and it has been successful to some extent in studies conducted under epidemiological conditions and adopting more sensitive diagnostic thresholds of dental caries ${ }^{1}$.

There has been underestimation of caries prevalence in surveys verified in groups of 12-year-old or in older children, when examined by the visual method ${ }^{2}$. In addition, the visual method of examination and WHO criteria have not been sufficient to properly identify the whole spectrum of the dental caries and 
subsequent treatment needs in posterior teeth ${ }^{3}$.

According to the systematic review of Bader et $a .^{4}{ }^{4}$, the strength of scientific evidence for adjunct methods of caries detection is poor. However, some studies have presented satisfactory results with the use of auxiliary resources (often low-tech and low-cost actions that improve visual examination as previous tooth brushing and drying or artificial lighting, for example) and adjunct methods (often high-tech devices as fiber-optic transillumination/FOTI, Diagnodent ${ }^{\mathrm{TM}}$, Electronic Caries Monitor/ECM or digital radiography, for example) for the detection of caries $^{5-6}$.

Apart from adding new diagnostic adjuncts with the aim of designing a more reliable nosological chart, more sensitive diagnostic thresholds recording the presence of initial carious lesions and/or activity have been proposed with some success in the literature $e^{1,3,7}$

Nowadays, epidemiological studies have shown that initial caries lesions in posterior teeth are very common. The detection of the whole spectrum of dental caries is an important tool for oral health services planning and evaluation. The aim of this study was to evaluate the performance of dental caries detection when adjunct methods are associated and their feasibility in epidemiological survey of dental caries, at D3 (cavitated carious lesions at dentin layer) and D1 + D3 (non cavitated and cavitated carious lesions at enamel/dentin layer) diagnostic thresholds.

\section{Materials and methods}

The study was conducted in accordance with resolutions 196/96 of National Health Council of Brazilian Ministry of Health, and resolution CFO 179/93 of the Dental Professional Code of Ethics, started after its approval by Research Ethics Committee Protocol No. 082/2006.

\section{Dental examinations}

The epidemiological data were gathered by clinical visual examination without (CL1) or with artificial lighting (CL2), radiographic bitewing examination (BW), FOTI (FT), laser fluorescence examination (DIAGNOdent ${ }^{\mathrm{TM}} / \mathrm{DD}$ ) and their respective associations: CL2FT, CL2DD, CL2BW, CL2FTDD, CL2FTBW, CL2DDBW and CL2FTDDBW. The codes and criteria $^{1,7-10}$ are shown in Table 1 .

Table 1. Diagnostic criteria and codes for dental examinations

\begin{tabular}{|c|c|c|c|c|c|c|c|}
\hline \multirow[b]{2}{*}{ Code } & CL1 and CL2 exams ${ }^{*}$ & \multicolumn{2}{|r|}{ Bitewing" } & \multirow[b]{2}{*}{ Code } & \multirow{2}{*}{$\begin{array}{l}\text { FOTr } \\
\text { Classification }\end{array}$} & \multicolumn{2}{|c|}{$\begin{array}{l}\text { DIAGNOdent }{ }^{\text {TM"* }} \\
\text { Code }\end{array}$} \\
\hline & Classification & Code & Classification & & & $\begin{array}{l}\text { Code } \\
\text { (reading } \\
\text { values) }\end{array}$ & Classification \\
\hline 0 & Sound & 0 & Absent surface & 0 & Absent surface & $0(0-10)$ & Sound surface \\
\hline IL & $\begin{array}{c}\text { Active initial enamel carious } \\
\text { lesions }\end{array}$ & $\mathrm{R} 1$ & Radiolucency in enamel & $\mathrm{F} 1$ & $\begin{array}{c}\text { Shading in } \\
\text { enamel }\end{array}$ & $1(11-20)$ & $\begin{array}{c}\text { Enamel carious } \\
\text { lesions }\end{array}$ \\
\hline 1 & Decayed & $\mathrm{R} 2$ & $\begin{array}{l}\text { Radiolucency in enamel (< } \\
1 / 2 \text { dentin thickness) }\end{array}$ & $\mathrm{F} 2$ & $\begin{array}{l}\text { Shading in } \\
\text { dentin }\end{array}$ & $2(21-99)$ & $\begin{array}{c}\text { Dentin carious } \\
\text { lesions }\end{array}$ \\
\hline $1 \mathrm{IL}$ & $\begin{array}{l}\text { Decayed + active initial } \\
\text { enamel carious lesions }\end{array}$ & R3 & $\begin{array}{l}\text { Radiolucency in dentin (> } \\
\qquad 1 / 2 \text { dentin) }\end{array}$ & 4 & Filled surface & & \\
\hline 2 & Filled, with decay & 4 & Filled surface & 5 & Sound surface & & \\
\hline $2 \mathrm{IL}$ & $\begin{array}{c}\text { Filled, with decay + active } \\
\text { initial enamel carious } \\
\text { lesions }\end{array}$ & 5 & Sound surface & & & & \\
\hline 3 & Filled & R6 & Impossible diagnosis & & & & \\
\hline 3IL & $\begin{array}{c}\text { Filled + active initial enamel } \\
\text { carious lesions }\end{array}$ & & & & & & \\
\hline 4 & Missing, as result of caries & & & & & & \\
\hline 5 & Missing, any other reason & & & & & & \\
\hline 6 & Sealant & & & & & & \\
\hline $6 I L$ & $\begin{array}{l}\text { Sealant + Initial enamel } \\
\text { carious lesions }\end{array}$ & & & & & & \\
\hline 7 & $\begin{array}{l}\text { Bridge abutment, special } \\
\text { crown or veneer/implant }\end{array}$ & & & & & & \\
\hline 8 & Unerupted tooth & & & & & & \\
\hline $\mathrm{T}$ & Trauma (fracture) & & & & & & \\
\hline 9 & Not recorded & & & & & & \\
\hline
\end{tabular}

* Adapted from Assaf et al. ${ }^{1}$, Fyffe et al. ${ }^{7}$ and $\mathrm{WHO}^{8}$

${ }^{* *}$ Adapted from Hintze et al. ${ }^{9}$

${ }^{* * *}$ Criteria and codes from Zanin et al. ${ }^{10}$ 
The CL1 and CL2 exams were carried out by using dental mirror, WHO probe, previous tooth brushing and air-drying for $5 \mathrm{~s}$ per each dental surface. The D3 diagnostic criterion, which considers cavitated carious lesions in dentine, and the D1 + D3 diagnostic criterion, which considers cavitated and non-cavitated carious lesions in enamel and/or dentine were adopted. Bitewings were taken of the schoolchildren's posterior teeth in a separate session, by an examiner experienced in this type of dental examination. The radiographical film Agfa Dentus M2 Comfort - M2-58, E/F-speed, and a Spectro 70X X-ray device (Dabi-Atlante, Ribeirão Preto, SP, Brazil) of 70 Kvp, $8 \mathrm{MA}$ and $0.4 \mathrm{~s}$ exposure time were used. Radiograph processing was standardized and performed in an automatic processor. The radiographs were analyzed in a viewing box (VH, Araraquara, SP, Brazil) without magnification by the benchmark examiner and the participant examiner. For the examination of proximal dental surfaces by FOTI, a FiberLite ${ }^{\circledR}$ PL-800 series device (Dolan-Jenner Elaborate, Lawrence, MA, USA), with a $0.5 \mathrm{~mm}$ diameter tip (positioned below the proximal point of contact) was used. The laser fluorescence examination by a DIAGNOdent ${ }^{\mathrm{TM}} 2095$ device (KaVo, Biberach, Germany) was performed according to the manufacturer's recommendations for the occlusal dental surfaces, but considering the classification scale and interpretation of the reading values proposed by Zanin et al. ${ }^{10}$, which was more adequate for the study proposals.

\section{Sample}

One hundred and sixty five 12-year-old schoolchildren from public schools in Piracicaba, SP, Brazil, with low to high prevalence of dental caries, were randomly selected. The sample size was determined considering an agreement proportion of $90 \%$, disagreement proportion of $5 \%$, test power of $92 \%$ and an alpha of 5\% under a bilateral test. Schoolchildren who presented fixed orthodontic devices, severe fluorosis and enamel hypoplasia or a serious systemic disease were replaced by other children without these conditions. Only the mesial, distal and occlusal dental surfaces of permanent posterior teeth were considered in this study, totaling 6565 surfaces of 2189 teeth. Two dental surfaces were excluded because they could not be examined by all the diagnostic methods.

\section{Calibration of examiner}

The calibration between the benchmark examiner and the participant examiner consisted of 9 sessions lasting $4 \mathrm{~h}$, contemplating lectures, clinical training and calibration exercises for CL1, CL2, BW, FOTI and DIAGNOdent ${ }^{\mathrm{TM}}$. A time interval of 10 days, needed to measure the intra-examiner agreement (only for the participant examiner), was adopted. Thirteen schoolchildren, with low to high caries prevalence, exclusively examined for this purpose, were involved in the final calibration exercise. The Cohen's Kappa statistics was used for both intra and inter-examiner agreement.

\section{Statistical analysis}

Sensitivity (Sn), specificity (Sp) and accuracy (A) tests were used to evaluate the validity of the dental caries examinations. The standard for the validation of exams was the CL2BW exam performed by the benchmark examiner. Each diagnostic method was compared with the CL2BW (performed by the benchmark examiner) by the McNemar test $(a=0.05)$. ANOVA was performed in order to compare the average values of DMF-S obtained by the diagnostic methods. The Scott-Knott test, which is used when there are too many treatment modalities, and there is interest to separate their results without ambiguity, was used for this purpose.

\section{Results}

The reproducibility was calculated by Cohen's Kappa statistics. At the D3 diagnostic criterion, the Kappa values for the inter-examiner agreement were 0.87 for CL1 and CL2, 0.94 for BW, 0.92 for FOTI, and 0.34 for DD exam, while the intra-examiner agreement was 0.99 for CL1 and CL2, 1.00 for $\mathrm{BW}$ and FOTI, and 0.42 for DD exam. At the D1+D3 diagnostic criterion, the Kappa values for inter-examiner agreement were 0.86 for CL1 and CL2, 0.88 for BW, 0.89 for FOTI and 0.48 for DD exam, while for the intra-examiner agreement the values were 0.98 for CL1, 0.97 for CL2, 1.00 for BW, 0.95 for FOTI and 0.42 for DD exam.

The validity of the dental caries examinations at the D3 and D1+D3 diagnostic thresholds are shown in the Table 2.

At both diagnostic thresholds the CL2FTDDBW exam presented the best values of sensitivity (Sn). However, the CL2BW exam produced a satisfactory clinical performance with good values of sensitivity ( $\mathrm{Sn}$ ) and accuracy (A) (Table 2).

The mean DMF-S index values as well as their differences among the dental caries examinations, measured by ANOVA (Scott-Knott test), are presented in Table 3.

At both diagnostic thresholds, the association of BW with the CL2 exam (CL2BW) was responsible for a considerable improvement in determining the DMF-S index. However, the simultaneous association of the BW and DD exams with the CL2 exam was statistically similar to the performance of CL2FTDDBW and differed statistically from the CL2BW performance for all diagnostic thresholds (Table 3).

\section{Discussion}

Diagnosis of dental caries has been considered a complex process, under both clinical and epidemiological conditions, due to the current pattern of disease, characterized by a smaller number and size of lesions and a concentration of them mainly in the posterior teeth ${ }^{2,11}$. A great number of studies deal with the development and the use of new technologies to improve the diagnosis of dental caries, especially at the early stages ${ }^{5,12-19}$. Generally, such studies are performed under controlled conditions, in vitro (laboratory settings) or in vivo (clinical settings) methodology. None was performed in epidemiological settings.

The first limitation of the present study was its "gold standard" to validate the diagnostic methods. Despite the 
Table 2. Validity of the dental caries examinations

\begin{tabular}{llll|lll}
\hline & \multicolumn{5}{c}{ Occlusal + approximal surfaces } \\
& \multicolumn{3}{c}{ D3 criterion } & \multicolumn{3}{c}{ D1+D3 criterion } \\
\hline Examinations & Sn & Sp & A & Sn & Sp & A \\
CL1 & 4.56 & 99.88 & $89.69^{*}$ & 56.61 & 98.61 & $91.21^{*}$ \\
CL2 & 4.99 & 99.88 & $89.73^{*}$ & 57.99 & 98.43 & $91.30^{*}$ \\
BW & 77.08 & 99.26 & $96.85^{\star}$ & 59.74 & 99.18 & $92.16^{*}$ \\
CL2FT & 5.13 & 99.86 & $89.74^{*}$ & 59.46 & 97.73 & $90.98^{*}$ \\
CL2DD & 33.48 & 97.99 & $91.09^{*}$ & 68.19 & 95.97 & $91.08^{*}$ \\
CL2BW* & 77.49 & 99.23 & $96.91^{*}$ & 86.78 & 97.69 & 95.76 \\
CL2FTDD & 33.62 & 97.97 & $91.08^{*}$ & 69.66 & 95.27 & $90.76^{*}$ \\
CL2FTBW & 77.49 & 99.22 & $96.90^{*}$ & 87.12 & 96.99 & 95.24 \\
CL2DDBW & 82.62 & 97.37 & 95.79 & 90.15 & 95.25 & $94.35^{*}$ \\
CL2FTDDBW & 82.62 & 97.36 & 97.78 & 90.49 & 94.55 & $93.83^{*}$ \\
\hline
\end{tabular}

* Significant difference between diagnostic method and validation method (pd"0.05).

** Performed by participant examiner. All examinations were tested against the CL2BW exam performed by the benchmark examiner

Table 3. DMF-S index values obtained by the examinations at the D3 and D1 +D3 diagnostic thresholds.

\begin{tabular}{lll|ll}
\hline Examinations & \multicolumn{2}{l|}{ D3 criterion } & \multicolumn{2}{l}{ D1+D3 criterion } \\
& DMF-S & SD & DMF-S & SD \\
CL1 & $1.32 \mathrm{a}$ & 2.45 & $6.58 \mathrm{a}$ & 4.97 \\
CL2 & $1.37 \mathrm{a}$ & 2.50 & $6.92 \mathrm{a}$ & 5.14 \\
BW & $4.55 \mathrm{c}$ & 2.17 & $6.88 \mathrm{a}$ & 3.74 \\
CL2FT & $1.39 \mathrm{a}$ & 2.51 & $7.67 \mathrm{a}$ & 8.02 \\
CL2DD & $3.29 \mathrm{~b}$ & 2.96 & $8.38 \mathrm{~b}$ & 4.98 \\
CL2BW** & $5.32 \mathrm{~d}$ & 2.58 & $9.06 \mathrm{~b}$ & 4.95 \\
CL2FTDD & $3.32 \mathrm{~b}$ & 2.99 & $8.68 \mathrm{~b}$ & 5.11 \\
CL2FTBW & $5.59 \mathrm{~d}$ & 2.56 & $9.00 \mathrm{~b}$ & 4.75 \\
CL2DDBW & $5.97 \mathrm{e}$ & 2.58 & $9.72 \mathrm{c}$ & 4.84 \\
CL2FTDDBW & $6,23 \mathrm{e}$ & 2.61 & $10.08 \mathrm{c}$ & 4.78 \\
\hline
\end{tabular}

Mean values followed by distinct letters on column-wise are statistically different $(p<0.05)$.

** Perfomed by participant examiner.

controversy around the association between visual (CL2) and bitewing radiography (BW) exams performed by an experienced examiner as "gold standard", such association was adopted. Ideally, the histological validation and the opening lesions are the most indicated methods to such purposes. However, those validation methods could not be adopted because the design of the present study simulates a real epidemiological survey of dental caries. Satisfactory results from the CL2 or CL2BW exams have been related in literature ${ }^{13,20}$, especially when immediate operative intervention is not implemented ${ }^{12,15}$. The exclusive use of bitewing radiographs as baseline in permanent dentition may be considered for populations with an overall low caries prevalence. That recommendation, along with the correctness of technique, the use of faster films, intraoral bitewing holders and leaded protective aprons with a thyroid collar, minimize the hazards of ionizing radiation ${ }^{21}$.

The purposes of an epidemiological survey of dental caries include registering the trend of the disease and the general treatment needs of a particular population ${ }^{8}$ aiming to organize the demand and the oral health services. For this reason, dental examinations at epidemiological survey are not suitable for caries diagnosis and treatment. To detect carious lesions is different from to diagnosing the dental caries disease. For caries diagnosis and subsequent decision treatment, a set of other factors must be considered. Such factors include the initial caries signs, the rest of the dentition, the patient's case history and population factors, fluoride availability and sugar intake. Diagnostic decision making is a balancing act ${ }^{5}$.

Although there is low strength to the evidence as regards the performance of diagnostic methods ${ }^{4}$, there are positive results described in the literature 2,6-7,22 $^{2}$ about the association of auxiliary resources and adjunct diagnostic methods for identifying initial carious lesions. This trend was evident and was in agreement with the results of this study. Although the poor reproducibility of the DD exam in epidemiological setting, it was maintained exclusively as an adjunct technique $^{22}$. Such reproducibility may be explained by the tooth cleaning method used in the study. The DIAGNOdent ${ }^{\circledR}$ manufacturer's advice is to perform professional prophylaxis previous to the examination. However, it is time-consuming and not required for visual examination. A previous tooth brushing (and drying procedure) is sufficient for that purpose. Nevertheless, toothpaste and biofilm remaining over occlusal dental surfaces may have influenced the DD readings ${ }^{23}$.

In the present study, the association of the clinical method with different adjunct technologies improved the sensitivity to detect caries, corroborating the results of Pereira et al. ${ }^{5}$. It is especially important to use methods or their association with others, which generate high sensitivity results in detecting caries in populations that present moderate to high prevalence of non-cavitated or hidden dentin lesions. Therefore, the sensitivity values of CL2DDBW and CL2FTDDBW at both diagnostic thresholds could be better indicated for these populations. However, since the CL2DDBW and CL2FTDDBW DMF-S means did not differ statistically, the use of CL2DDBW could be suggested first, assuming that such examination is simpler to perform than the CL2FTDDBW. 
On the other hand, CL1, CL2 and CL2FT at the D3 diagnostic threshold, and CL1, CL2 and BW at the D1+D3 diagnostic threshold, would be better indicated in populations with low caries prevalence due to the high specificity of their results. Since the DMF-S means did not differ statistically, CL1 and CL2 at both diagnostic thresholds could be recommended in surveys, although the most accurate methods were CL2FTDDBW at the D3 diagnostic threshold and CL2BW at the D1+D3 diagnostic threshold.

Although the DMF-S obtained by CL2BW is statistically different from the values from CL2DDBW and CL2FTDDBW (Table 3), its results (Tables 2 and 3) cannot be neglected due its availability and familiarity among dentists. The purposes of a dental caries surveys support such differences.

Furthermore, CL1 and CL2 accuracy results at the D1 + D3 diagnostic threshold, indicated that a well-trained and competent professional is able to detect caries lesions. Therefore, training strategies should be focused not only on private but also on public services, because professional experience may influence the diagnosis of caries ${ }^{5}$ and treatment of the disease, which is often underestimated by professionals $^{24}$.

Low sensitivity values for CL1 and CL2 methods, at the D3 threshold, could be explained by the fact that dentin lesions under intact enamel are rarely detected without the use of an adjunct method, mainly the bitewing radiographic method. In addition, results are sometimes compromised by the examiners' subjectivity due to their previous clinical experience, verified even among calibrated examiners, such as those who conducted this survey. An improvement in the sensitivity of CL1, CL2 and BW, with the association of DIAGNOdent $^{\mathrm{TM}}$ was found in this survey. That improvement in sensitivity is followed by a decrease in specificity. This is critical in populations whose caries prevalence is low, increasing the probability of false positive diagnoses ${ }^{5,12,21}$. In spite of its performance in non-cavitated caries lesions on occlusal surfaces ${ }^{13,19}$, use of DIAGNOdent ${ }^{\mathrm{TM}}$ in epidemiological surveys might be not recommended mainly due to its high cost. It is important to mention that improvement in detecting caries with the DIAGNOdent ${ }^{\mathrm{TM}}$ could be also explained by its trend to produce false-positive diagnoses ${ }^{4}$. According to Pereira et al. ${ }^{5}$, to have data available from multiple methods may influence on the number of surfaces indicated for operative treatment. So, the adoption of diagnostic adjuncts in epidemiological surveys must be only used to supplement the decision to plan preventive strategies for initial dental caries ${ }^{16}$.

Nowadays, professionals should be more prepared to diagnose properly a higher number of intact teeth or teeth with initial caries, than to detect cavitated lesions in dentin, due to the current less progressive pattern of disease. For this reason, it is recommended to "wait and watch" and not to immediately "drill and fill" teeth ${ }^{25}$. According to Novaes et al. ${ }^{17}$, visual inspection alone seems to be sufficient to detect dental caries satisfactorily. The results from CL1 and CL2 exams corroborate that statement in some degree. The training for dental caries diagnosis under a more refined threshold is also needed nowadays ${ }^{12}$. However, studying alternatives to the traditional diagnostic methods and criteria does not mean to substitute them in all circumstances. The availability to acquire such diagnostic adjuncts and subsequent opportunity to apply them must always be considered. The WHO criteria $^{8}$ still have their important role in Dentistry.

It is also relevant to know the advantages and disadvantages of diagnostic technologies and their associations, because there is no modality of examination that can be successfully used alone for diagnosing caries and able to produce representative data of the disease in a population $^{5,14}$.

In conclusion, the association of adjunct diagnostic methods with the clinical method generated higher validity results than the clinical method alone, at both D3 and D1 + D3 diagnostic thresholds, under epidemiological examination conditions. Diagnosing is a balancing act. Therefore, considering the familiarity of the professionals with clinical and radiological examinations, the present results show the potential of the CL2BW in epidemiological surveys of dental caries. The visual exam (CL1 and CL2), under more refined diagnostic criteria, also showed its potential at epidemiological surveys. However, studies with similar methodologies are needed to endorse the association of adjunct diagnostic methods in epidemiological settings.

\section{Acknowledgement}

The authors would like to thank the volunteers and those responsible for the schoolchildren of the public schools in Piracicaba, SP, Brazil. The first author received a scholarship from PROEX/CAPES-UNICAMP during the Doctorate Course in Dentistry. The authors acknowledge the financial support of the FAPESP (grants \#06/58881-9).

\section{References}

1. Assaf AV, Meneghim MC, Zanin L, Mialhe FL, Pereira AC, Ambrosano GMB. Assessment of different methods for diagnosing dental caries in epidemiological surveys. Community Dent Oral Epidemiol. 2004; 32: 418-25.

2. Hopcraft MS, Morgan MV. Comparison of radiographic and clinical diagnosis of approximal and occlusal dental caries in a young adult population. Community Dent Oral Epidemiol. 2005; 33: 212-8.

3. Kühnisch J, Berger S, Goddon I, Senkel H, Pitts N, Heirinch-Weltzien R. Occlusal caries detection in permanent molars according to WHO basic methods, ICDAS II and laser fluorescence measurements. Community Dent Oral Epidemiol. 2008; 36: 475-84

4. Bader JD, Shugars DA, Bonito AJ. Asystematic review of the performance of methods for identifying carious lesions. J Public Health Dent. 2002; 62 201-13.

5. Pereira AC, Eggertsson H, Martinez-Mier EA, Mialhe FL, Eckert GJ, Zero DT. Validity of caries detection on occlusal surfaces and treatment decisions based on results from multiple caries-detection methods. Euro J Oral Sci. 2009; 117: 51-7.

6. Souza-Zaroni WC, Ciccone JC, Souza-Gabriel AE, Ramos RP, Corona SAM, Palma-Dibb RG. Validity and reproducibility of different combinations of methods for occlusal caries detection: an in vitro comparison. Caries 
Res. 2006; 40: 194-201.

7. Fyffe HE, Deery C, Nugent ZJ, Nuttall NM, Pitts NB. Effect of diagnostic threshold on the validity and reliability of epidemiological caries diagnosis using the Dundee Selectable Threshold Method for caries diagnosis (DSTM). Community Dent Oral Epidemiol. 2000; 28: 42-51.

8. World Health Organization (WHO). Oral health surveys: basic methods. 5th ed. Geneva: WHO; 2013.

9. Hintze $H$, Wenzel A, Danielsen B, Nyvad B. Reliability of Visual Examination, Fibre-Optic Transillumination, and Bite-Wing Radiography, and Reproducibility of Direct Visual Examination Following Tooth Separation for the Identification of Cavitated Carious Lesions in Contacting Approximal Surfaces. Caries Res. 1998; 32: 204-9.

10. Zanin L, Meneghim MC, Assaf AV, Pardi V, Pereira AC, Mialhe FL. Depth of occlusal caries assessed clinically by fluorescence laser, conventional and digital radiographic methods. Braz J Oral Sci. 2005; 4: 735-40.

11. Marthaler TM. Changes in dental caries 1953-2003. Caries Res. 2004; 38: 173-81.

12. Baelum V, Hintze H, Wenzel A, Danielsen B, Nyvad B. Implications of caries diagnostic strategies for clinical management decisions. Community Dent Oral Epidemiol. 2012; 257-66.

13. Diniz MB, Boldieri T, Rodrigues JA, Santos-Pinto L, Lussi A, Cordeiro $\mathrm{RCL}$. The performance of conventional and fluorescence-based methods for occlusal caries detection: an in vivo study with histologic validation. J Am Dent Assoc 2012; 143: 339-50.

14. Goel A, Chawla HS, Gauba K, Goyal A. Comparison of validity of DIAGNOdent with conventional methods for detection of occlusal caries in primary molars using the histological gold standard: an in vivo study. J Indian Soc Pedod Prevent Dent. 2009; 27: 227-34. doi: 10.4103/09704388.57658.

15. Huth KC, Neuhaus KW, Gygax M, Bücher K, Crispin A, Paschos E, et al. Clinical performance of a new laser fluorescence device for detection of occlusal caries lesions in permanent molars. J Dent. 2008; 36: 103340. doi: 10.1016/j.jdent.2008.08.013.

16. Khalife MA, Boynton JR, Dennison JB, Yaman P, Hamilton JC. In vivo evaluation of DIAGNOdent for the quantification of occlusal dental caries. Oper Dent. 2009; 34: 136-41. doi: 10.2341/08-54.

17. Novaes RF, Matos R, Gimenez T, Braga MM, Benedetto MS, Mendes FM. Performance of fluorescence-based and conventional methods of occlusal caries detection in primary molars - an in vitro study. Int J Paediatr Dent. 2012; 22: 459-66. doi: 10.1111.

18. Rechmann P, Charland D, Rechmann BMT, Featherstone JDB. Performance of laser fluorescence devices and visual examination for the detection of occlusal caries in permanent molars. J Biomed Opt. 2012; 17: 036006. doi: $10.1117 / 1$.

19. Souza JF, Boldieri T, Diniz MB, Rodrigues JA, Lussi A, Cordeiro RCL. Traditional and novel methods for occlusal caries detection: performance on primary teeth. Laser Med Sci. 2013; 28: 287-95. doi: 10.1007/s10103012-1154-8.

20. Wenzel A. Bitewing and digital bitewing radiography for detection of caries lesions. J Dent Res. 2004; 83 Spec No C: C72-5.

21. Espelid I, Mejàre I, Weerheijm K. EAPD guidelines for use of radiographs in children. Eur J Paediatr Dent. 2003; 4: 40-8.

22. Bader JD, Shugars DA. A systematic review of the performance of a laser fluorescence device for detecting caries. J Am Dent Assoc. 2004; 135: 1413-26.

23. Lussi A, Reich E. The influence of toothpastes and prophylaxis pastes on fluorescence measurements for caries detection in vitro. Eur J Oral Sci. 2005; 113: 141-4.

24. Traebert J, Marcenes W, Kreutz JV, Oliveira R, Piazza CH, Peres MA. Brazilian dentist's restorative treatment decisions. Oral Health Prev Dent. 2005; 3: 53-60.

25. Bader JD, Shugars DA. The evidence supporting alternative management strategies for early occlusal caries and suspected occlusal dentinal caries. J Evid Based Dent Pract. 2006; 6: 91-100.

Braz J Oral Sci. 14(1):78-83 